\title{
Efect of spatially variable effective mass on static and dynamic properties of resonant tunneling devices
}

\author{
R. K. Mains, 1. Mendi, and G. I. Haddad \\ Center for High-Frequency Microelectronics, Department of Electrical Engineeping and Computer Science, \\ The University of Michigan, Ann Arbor, Michigan 48109
}

(Received 3 July 1989; accepted for publication 11 October 1989)

The effect of incorporating a spatially variable effective mass in the Schrödinger equation method of resonant tunneling device modeling is investigated. It is shown that inclusion of this effect can produce an order of magnitude difference in the calculated peak current density of the static current-voltage $(X-W)$ curve for the resonant tunneling diode. Results for a particular $\mathrm{In}_{0.53} \mathrm{G}_{a_{0.47}}$ As-AlAs structure show that much better agreement between theory and experiment is obtained by including this effect. Also, comparison of transient results for an $\mathrm{In}_{0.53} \mathrm{Ga}_{0.47} \mathrm{As}-\mathrm{In}_{0.52} \mathrm{Al} \mathrm{l}_{0.48}$ As structure shows a significant change in the diode switching transients.

The state of the art of resonant tunneling diode modeling is such that good agreement between calculated and experimental results is difficult to obtain. Analyses using energy eigenstates obtained from the time-independent Schrödinger equation typically underestimate the bias voltage at which peak current occurs and overestimate the peak-to-valley current ratio. ${ }^{1-4}$ Wigner function calculations present severe numerical difficulties that have not yet been overcome. ${ }^{6}$

In this letter, the effects of including different effective masses in the Schrödinger equation corresponding to the different material layers of the device are investigated. The method of including variabie effective mass is to maintain continuity of $\left(1 / m^{*}\right) \partial \psi / \partial x$ in the finite difference approximation of Schrödinger's equation, which maintains current continuity within the envelope function approximation. 5,6 (To simplify the calculations, effective mass variations in the $x$ direction only are included, so that variations in transverse energy do not appear for energy eigenstates.7) This method is expected to work well when smoothly matched envelope functions are appropriate boundary conditions. However, for large discontinuities in effective mass a more complex treatment may be required. ${ }^{8,9}$ Results for $\mathrm{In}_{0.53} \mathrm{Ga}_{0.47} \mathrm{As}-\mathrm{AlAs}$ and $\mathrm{In}_{0.53} \mathrm{Ga}_{0.47} \mathrm{As}-\mathrm{In}_{0.52} \mathrm{Al}_{0.48} \mathrm{As}$ structures are presented.

The time-independent Schrödinger equation including spatially variable effective mass is taken to be

$\frac{-\hbar^{2}}{2} \frac{\partial}{\partial x}\left(\frac{1}{m^{*}(x)} \frac{\partial \psi(x)}{\partial x}\right)=(E-V(x)) \psi(x)$.

The current density for the quantum state $\psi$ is given by

$J(x)=-q^{2} \operatorname{Im}\left(\psi^{*}(x) \frac{1}{m^{*}(x)} \frac{\partial \psi(x)}{\partial x}\right)$.

Multiplying Eq. (1) by $\psi^{*}$ and subtracting the complex conjugate of the resulting equation, it can be shown that a solution $f(x)$ of Eq. (1) will have $J(x)$ constant with $x$. To preserve current continuity in the discrete form of the equation, the following finite difference form of $\mathrm{Eq}$. (1) is used:

$$
\frac{-\hbar^{2}}{2}\left(\frac{\left(1 / m_{i}^{*}\right)\left(\psi_{i+1}-\psi_{i}\right) / \Delta x-1 /\left(m_{i-1}^{*}\right)\left(\psi_{i}-\psi_{i-1}\right) / \Delta x}{\Delta x}\right)=\left(E-V_{i}\right) \psi_{i}
$$

where $i$ refers to meshpoint $i$. In Eq. (3), effective mass value $m_{i}^{*}$ is taken to be the effective mass midway between meshpoints $i$ and $i+1$, so that the effective mass values are specified on a staggered mesh with respect to wave function and potential energy values.

Collecting terms yields the following discrete equation for advancement to the right:

$$
\begin{aligned}
\psi_{i+1}= & \left(\frac{2 m_{i}^{*} \Delta x^{2}\left(V_{i}-E\right)}{\hbar^{2}}+1+\frac{m_{i}^{*}}{m_{i-1}^{*}}\right) \psi_{i} \\
& -\frac{m_{i}^{*}}{m_{i-1}^{*}} \psi_{i \ldots 1}
\end{aligned}
$$

or for advancement to the left:

$$
\begin{aligned}
\psi_{i, 1}= & \left(\frac{2 m_{i-1}^{*} \Delta x^{2}\left(V_{i}-E\right)}{\hbar^{2}}+1+\frac{m_{i-1}^{*}}{m_{i}^{*}}\right) \psi_{i} \\
& -\frac{m_{i-1}^{*}}{m_{i}^{*}} \psi_{i+1} .
\end{aligned}
$$

Using Eqs. (4) or (5), it can be shown that constant current is maintained if the following discrete expression for current density is used:

$$
\begin{aligned}
J_{i}= & -q \hbar \operatorname{Im}\left[\frac { \psi _ { i } ^ { * } } { 2 } \left(\frac{1}{m_{i}^{*}} \frac{\psi_{i+1}-\psi_{i}}{\Delta x}\right.\right. \\
& \left.\left.+\frac{1}{m_{i-1}^{*}} \frac{\psi_{i}-\psi_{i-1}}{\Delta x}\right)\right] .
\end{aligned}
$$


Equations (4) and ( 5 ) may be regarded as extensions of the equations given in Ref. 3 for the uniform effective mass case. A similar modification is made to the spatial derivative term in the time-dependent equation to calculate the transient response.

Figure 1 (a) shows the experimental dc current-voltage $(1-V)$ curve for a $24 \AA$ AlAs barrier- $44 \AA \ln _{0.53} \mathrm{Ga}_{0.47} \mathrm{As}$ well pseudomorphic structure at room temperature; the experimental peak current density is $-15 \mathrm{kA} / \mathrm{cm}^{2}$. These devices were grown by molecular beam epitaxy at a temperature of $500^{\circ} \mathrm{C}$ and a growth rate of $0.6 \mathrm{~mm} / \mathrm{h}$. Mesa diodes were fabricated using conventional lithography techniques. Nonalloyed onmic contacts of $\mathrm{Ni} / \mathrm{Ge} / \mathrm{Au} / \mathrm{Ti} / \mathrm{Al}$ were used. $^{10}$

Figure 1 (b) shows a calculated $I-V$ curve for which a uniform effective mass of $m^{*}=0.042 m_{0}$, the $\ln _{0.53} \mathrm{Ga}_{0.47} \mathrm{As}$ value, was used. In this calculation the assumed barrier discontinuicy was the $\Gamma$ value of $1.2 \mathrm{eV}$. Undoped spacer layers of $50 \AA$ were placed adjacent to each barrier, and concentrations outside the double-barrier structure were calculated using the Thomas-Fermi method. "Even with such a large barrier discontinuity, the calculated peak current density of
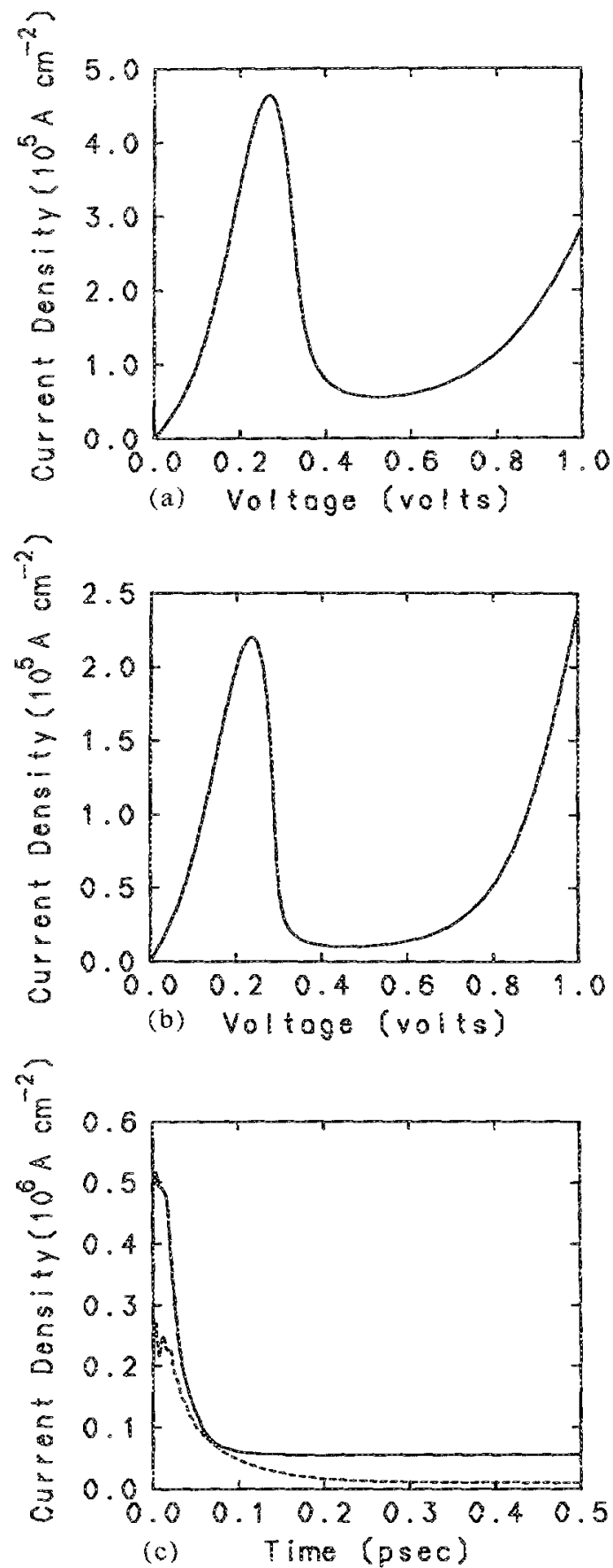

FrG. 2. $I . V$ curve calculated for $23 \AA \ln _{0 \$_{2}} \mathrm{Al}_{0.48}$ As barrier-44 $\AA$ In $n_{0.3,} \mathrm{Ga}_{0.4}$ As well structure (a) with unifom $m^{*}=0.42 m_{0}$, (b) using $m^{*} \ldots 0.075 m_{0}$ in the harriers, and (c) comparison of turnot transients with uniform (solid curve) and variable (dashed curve) effective mass. 
$357 \mathrm{kA} / \mathrm{cm}^{2}$ is over an order of magnitude larger than the experimental value. Figure l(c) shows a calculation with $m^{*}=0.15 m_{0}$, the bulk value for AlAs, used in the barrier regions and $m *=0.042 m_{0}$ outside. The effective mass discontinuity decreases the calculated peak current density to $9.3 \mathrm{kA} / \mathrm{cm}^{2}$, much closer to the experimental value. Since it is unrealistic to assume that the effective mass changes across a single meshpoint to the bulk AlAs value as is done for the results in Fig. I (c), it is expected that this calculation underestimates the peak current density. The current peak10-valley ratio is increased from 65 in Fig. 1 (b) to 3120 in 1 (c); the fact that phonon and electronelectron scattering are ignored in the quantum mechancal calculation for current may explain why the calculated peak-to-valiey ratios are much higher than the experimental value of 24 in $l$ (a).

The overall effect of introducing variable effective mass observed by comparing Figs. 1 (b) and 1 (c) is similar to the effect expected from increasing the barrier height for the uniform effective mass case. This behavior was pointed out in Ref. 12 from a study of the tunneling transmission coeffcient in the GaAs-Al $\mathrm{Ga}_{1-x}$ As material system. Increasing the barrier height is expected to reduce the peak current density, increase the current peak-to-valiey ratio, and decrease the device switching speed. ${ }^{3}$

Figure 2 (a) shows the static $l-V$ curve calculated for a $23 \AA \operatorname{In}_{0.52} \mathrm{Al}_{0.48}$ As barrier-44 $\AA \mathrm{In}_{0.53} \mathrm{Ga}_{0.47}$ As well structure assuming a uniform effective mass of $m^{*}=0.042 m_{0}$, the bulk $\ln _{0,53} \mathrm{Ga}_{0.47}$ As value. Figure $2(\mathrm{~b})$ shows the result using variable mass with $m^{*}=0.075 m_{0}$ in the barriers. (Since time-dependent calculations are also performed for this structure, the self-consistent potential is not included in these results.) In this case the assumed barrier discontinuity is $0.53 \mathrm{eV}$, less than half the discontinuity for the structure of Fig. 1. In addition, the effective mass discontinuity is smaller. $\mathrm{Bt}$ is seen that the reduction in peak current density is smaller for this case, al though a factor of 2 is still obtained. Figure $2(c)$ compares the switching transients from peakto-valley current points for the uniform (sclid curve) and variable mass (dashed curve) cases. (The initial oscillations are due to reflections between the barriers of electrons inside the quantum well. ${ }^{3}$ ) As expected, a decrease in switching speed is observed when the effective mass discontinuity is included. (Since this method does not include scattering and underestimates the valley current, it is expected that the actual reduction of switching speed is less.) This effect should become more important as the effective mass discontimuity increases.

A method of including variable effective mass in the solution of the time-independent and time-dependent Schrödinger equations within the envelope function approximation for resonant tunneling diodes has been presented. It was shown that inclusion of this effect is important for accurately predicting peak current densities and switching speeds for these devices.

This work was supported by the U.S. Amy Research Offee under the URE program, contract No. DAAL03-87$\mathrm{K} \cdot 0007$.

'W. R. Fransley, Phys, Rev. B 36, 1570 (1987).

2W. R. Fronsley, Phys. Rev. Lett. 60, 1589 (1988).

${ }^{3}$ R. K. Mains and G. I. Haddad, J. Appl. Mhys. 64, 3564 (1988).

4. K. Mains and G. I. Haddad, J. Appl. Phys. 64, 504 i (1988)

'D. J. BenDaniel and C. B. Duke, Fhys. Rev. 152,683 (1966).

G. Bastard, Rhys. Rev, Is 24,5693 (1981).

'M. O. Vassell, J. Lee, and H. F. Lockwood, J. Appl. Phys. 54, 5206 (1983).

1. Ando and S. Mori, Surf. Sci. 113, 124 (1982).

${ }^{9}$ Q. -G. Zhu and H. Kroener, Phys. Rev. B 27, 3519 (1983).

10. Mchdi and $G$. L. Haddad (unpubhished).

${ }^{11}$ R. K. Mains, J. P. Sun, and G. I. Haddad, Appl. Phys. Lett. 55, 371 (1989).

'K. F. Brennan and C. J. Summers, J. App!. Phys. 61, 614 (1987). 